\title{
Portraits of HIV: a pilot photovoice study of adolescent experiences with HIV treatment in South Africa
}

\author{
Brian van Wyk', Michelle Teti ${ }^{2}$ \\ 1 University of the Western Cape, School of Public Health, Cape Town, South Africa, ${ }^{2}$ University of Missouri Department of Public Health, School of \\ Health Professions, Columbia, Missouri, MO 65201, United States \\ Keywords: adolescent health, adherence, hiv, pilot study, qualitative research, photovoice \\ https://doi.org/10.29392/001c.12588
}

\section{Journal of Global Health Reports}

Vol. 4, 2020

\section{Background}

Adolescents are one of the fastest growing groups of people living with HIV (PLWH) in South Africa. They are also more likely than PLWH in other age groups to report poor adherence to antiretroviral therapy (ART). This trend is likely due to a confluence of factors including life stage and transition from child to adult care. Solutions are urgently needed to support adolescent adherence, since ART can improve the lives of PLWH and limit HIV transmission. We explored adolescents' facilitators and barriers to ART adherence via photovoice, to pilot the method with South African youth and gain information to inform future interventions.

\section{Methods}

The pilot study included three Photovoice groups, with 10 adolescents, who were enrolled in ART at two urban public primary health care centres in the Western Cape province of South Africa. Groups were organized by age and sex (group 1: 10-14 years, girls; group 2: 15-19 years, girls; group 3: 15-19 years, boys). Participants were given cell phones with cameras, and instructed to take pictures representing their experiences with HIV and treatment.

\section{Results}

Youth's pictures and discussions focused on four key themes related to treatment and ART adherence. These themes included motivations for taking medicine, forming an identity with HIV, managing disclosure and treatment, and relationships between living conditions and HIV treatment. Treatment motivation was informed by familial support. Themes shed light on participants' challenges and resilience in the face of HIV.

\section{Conclusion}

Adolescents found expressing their views through photos 'exciting' and were encouraged to share personal meanings in discussion with peers and researchers. Older adolescents needed less direction and encouragement than younger adolescents, to fully participate in photo-taking and discussions. Findings pave the way for future, larger Photovoice studies. Themes reiterate the importance of personal motivation including aspects of positive identity with HIV in helping youth adhere to medicine, as well as the need to address relational (e.g. family, disclosure) and structural (e.g. living conditions) factors in adherence interventions for this age group. Despite challenges, youth also have strengths (e.g. desire to commit to treatment) that interventionists can capitalize on to support adolescent treatment outcomes.

South Africa has the largest paediatric and adult antiretroviral therapy (ART) programme in the world, and an estimated 310,000 adolescents living with HIV (ALHIV) in 2018. ${ }^{1}$ The tremendous successes in paediatric HIV treatment has resulted in increased survival rates for perinatally infected children whom are now a rapidly growing sub-population of adolescents living with HIV (ALHIV). ${ }^{2}$ Adolescence - defined as 10-19 years - is a unique developmental period that is characterised by psychological and physiological changes and therefore requires age-appropriate care and treatment. Around the ages of 12-14 years, children with HIV transition from paediatric to adult HIV care, because adult care has superior treatment options. ${ }^{3}$ This transition is a high-risk period for disengagement from care as ALHIV experience the disruption of the patient-paediatric provider relationship and the loss of ancillary sup- 
port. ${ }^{4,5}$ They are also required to be independent and exercise greater autonomy and responsibility for managing their chronic HIV. The barriers to effective transition of ALHIV are numerous, and include mental health issues, stigma and lack of social support at home and school, amongst others. ${ }^{6,7}$

AIDS-related deaths among adolescents worldwide are increasing, while decreasing for all other age groups. The increase in mortality can be attributed to poor adherence and lower rates of viral suppression. ${ }^{1,8}$ As such, there is a growing body of research exploring the reasons behind poorer treatment outcomes among ALHIV. The patient level factors that influence adherence to treatment amongst adolescents on ART include mental health and well-being, nature of disclosure of HIV status, and duration on treatment as well as mode of HIV acquisition (behavioural vs perinatal). ${ }^{9-11}$

Adherence behaviour is dynamic and contextual; and influencing factors go well beyond patient factors alone. ${ }^{12,13}$ An in-depth exploration of the conditions that support and detract from adherence among adolescents is critical to develop and provide responsive HIV services for ALHIV. We conducted a pilot Photovoice study with adolescents aged 10-19 years, accessing treatment at two public primary health care facilities in the Western Cape Province of South Africa, to gain insights about adolescents' challenges and facilitators adhering to their medicine.

\section{METHODS}

\section{PARTICIPANTS}

This research was approved by the primary author's institutional review board. Participants were recruited from clinics that operated HIV adherence clubs for youth and adolescents ages 10-19 years. The research team met with key staff in the clinics to explain and discuss the project. Staff helped to recruit eligible participants or participants who were HIV positive, prescribed and/or taking anti-retroviral medication, aged 10-19 years, and able and willing to take part in project activities.

\section{PROCEDURES}

We used a Photovoice methodology to gather information from participants about their strengths and challenges related to taking HIV medication. Photovoice is a participatory research design in which participants use photographs to identify, capture, discuss, and share their ideas with their peers and their communities. ${ }^{14}$ We chose Photovoice because we wanted to give youth an alternate way, to words alone, to express their experiences. We believed that pictures would help youth creatively express difficult and complex experiences across language and literacy barriers. Because youth take pictures in their home and social environments, we believed Photovoice could also help shed light on changing and contextual factors that affected adherence. We also chose Photovoice because, as a participatory method, it would result in both data collection and opportunities for youth. For example, adolescents in the project would learn to use a camera, have an opportunity to discuss and share their ideas with other young people living with
HIV, and influence community members with photo sharing and exhibits. By their concrete nature, photos can be shared as a point of education, discussion, and ultimately, action.

In this article we describe the results of piloting Photovoice with ten ALHIV. Participants attended three sessions. In the first session, two study staff summarized the study and reviewed the study consent. During the first session, participants also met each other and shared information about their stories and situations, to facilitate group trust and bonding. Then participants left to discuss the study with their parents and attain parental permission. In session two, study staff explained the study in more detail to all participants who consented to take part in the study. They instructed participants to tell their story of taking HIV medicines, focusing on the strengths and challenges of taking medicine, with photos. They gave each participant a camera, taught participants to use the camera, and led a discussion to make sure participants had a plan to move forward. Participants helped each other generate ideas if needed. In the third session, the participants took turns sharing and discussing the meaning of their picture. The facilitators asked the participants the following questions: What is this a picture of? Tell us more about what it means to you. What were you thinking about when you took it? What does the picture say about your life with HIV and/or taking HIV medication? Probes explored issues that came up around the pictures in more details. After an individual shared their picture, the facilitators asked the group to react or respond to the picture, or group problem-solve challenges that arose in the picture presentation. This gave youth opportunities to learn from and support each other as they realized their differences and similarities. This aspect of Photovoice is critical to facilitating empowerment - or greater awareness of and control over problems and solutions.

\section{ANALYSIS METHODS}

We analyzed the data using strategies of theme analysis, to capture major ideas across and within participant stories. ${ }^{15}$ The first author, who also co-facilitated the sessions, and the second author reviewed every transcript and created a list of key themes and topics arising in the data independently. Then, they discussed their findings and narrowed the themes down to five major categories. The two authors defined these themes together, and created a codebook. The first author coded the data using the codebook, meeting regularly with the second author to discuss and address coding challenges or discrepancies. The second author created a narrative table that outlined each participant, their photos, and the stories they told around each photo. Using printouts of the coding report and the narrative table, the authors wrote the results section.

\section{RESULTS}

Ten participants were recruited from two primary health care clinics in the Western Cape Province in South Africa. The final sample included nine adolescents; one participant did not return for the third session and could not be contacted. From this sample we conducted three different Photovoice projects; the first consisted of two girls both aged 10 
years, the second group consisted of four boys aged 15-17 years, and the last group consisted of four girls aged 15-17 years. All participants were infected with HIV perinatally. Although all participants understood and were able to converse in English, only one of the participants spoke English as a first language. The majority of participants $(n=6)$ spoke isiXhosa as their first language and two of the participants identified Afrikaans as their first language. The analysis of the transcripts and photos revealed five main themes that summarized participants' lives with HIV and treatment experiences: motivations and prompts to take medications; identity with HIV; disclosure of HIV status; and living conditions. Below we describe each theme in more detail with participant quotations and photos. Pseudonyms replace participant's names.

\section{MOTIVATIONS AND PROMPTS TO TAKE MEDICINE}

Given that this project focused on treatment, medication practices were, not surprisingly, a key theme. All the participants in this study were on treatment since early childhood. Most were on the adult ART regimen. They reported high adherence to their medicine, and discussed what prompted or motivated them to take their medicines. Bernadette (girl, 15 years) showed pictures of water and of fruit and vegetables (Figure 1), indicating that living in a healthy home with access to water and nutritious food motivated her to take her HIV medication. During this conversation she also pointed out that her father always supported her needs, saying,

"I'm grateful because I live in a house. There's always
clean water, and even when the water is off my daddy
always has a spare bottle of water."

Photos like this spurred conversations of parents' roles in medication adherence. Many parents were key in supporting their children's medication routines. Like Lulu (boy 15), participants said parents helped by

"He tells me which time I must go out and which time I must come back to take my medication."

Tuli (boy, 15) said his mother was "on his side" because she also had HIV, and tells him "when it is the time" to take medication. Ziya (girl 10) also indicated that she and her mother reminded each other when to take medicines.

Parents sometimes used negative reinforcement, however. Two children noted that their parents warned the children they would die if they did not take medicine regularly. Busi (girl 10) said,

\footnotetext{
"She say I must take this because the med- the treatment because...if I don't take the meds, I will...I will die or be sick."
}

Tuli (boy, 15) noted that,

$$
\mid \begin{aligned}
& * \text { “'I motivate myself by telling myself that...If I don’t do } \\
& \text { that I will die.” }
\end{aligned}
$$

Ziya's mother told her she could be sent away if she did not take medicines. Participants did not report being scared by these statements. Busi (age 10) noted that these mes-

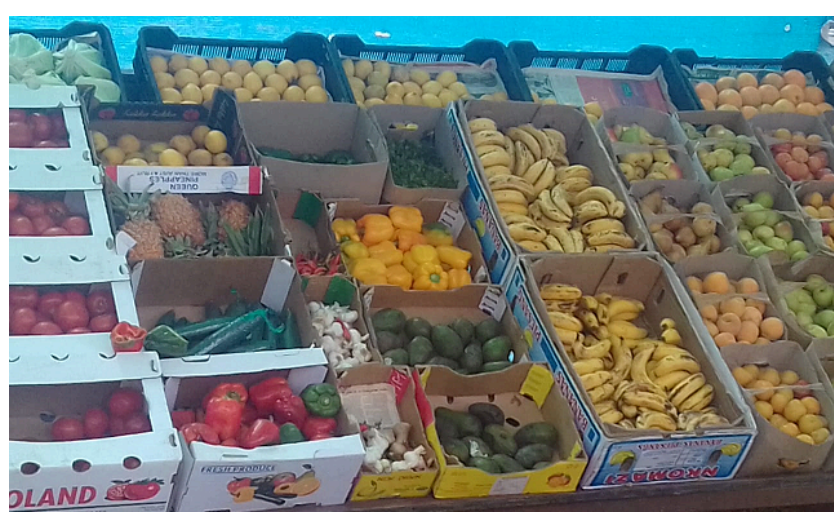

Figure 1. Fruits and vegetables.

sages did not make her

"feel sad because she [mother] is making me powerful
to know that if I am not taking my treatments, what will
happen to me."*

The older adolescent girls worked out reminders for themselves to take their medication. They were all on a onea-day adult ART regimen (fixed dose), and all three said they used the daily TV "soapies" as reminders. One participant shared a picture of the show on the television screen to make this point:

"My second picture is about my favourtie soapie...It's the same time I come out of church every evening and it's the perfect time for me to take my medicine."

Hashi shared a picture of her evening meal to show what prompted her to take her pills, saying,

" The reason why I took that picture is because before I drink my tablets, I always eat my vegetables."

\section{IDENTITY WITH HIV}

Participants also captured and discussed their identity with HIV via pictures and group conversations. Most of the participants reported that they accepted their HIV status and were "happy" to take medication because it was simply part of their lives now. For instance, Tumi (boy, 16), said,

\section{"I feel happy because...without my medication I wouldn't be here...and speaking right now."}

Lulu (boy, 15) demonstrated that he was thriving with HIV with a picture of a tree:
"The tree is a good example of a human being, because it starts from the seed and [grows to a human adult]...the tree here tells me that if a human being continues to take his medication, he will grow up and feel free...when a human being...takes care of his body... [he] will be strong, fresh, energetic...and feel good for their life."

However, Lulu also expressed that although he lives positively, and describes himself as a "HIV survivor", he still has questions about "why he has HIV", as illustrated in the picture (Figure 2) that he took of the heart. He explained his 
conflicted emotions, saying that he knows he "has a good heart", but then "thinks about this whole 'I am living with HIV" " and questions his health.

Several participants associated their identity with HIV with the need to take care of themselves. The older girls discussed and shared photos of tobacco and substance use. Bernadette shared a picture of an 'okka pipe' (Hookah pipe) to illustrate that she needs to make decisions that are in the interest of her health.

\section{"HIV is in your blood stream and okka pyp isn't healthy for your system...sooner or later your lungs will be col- lapsing. And what happens to your body? Your body is weak. You have to take your medicine to feel better and leave the okka pyp alone."}

Similarly, another girl in the group (Moya 15) showed a picture of a cigarette titled, “Don't smoke cigarettes”, and made a similar point about the importance of being healthy.

\section{DISCLOSURE OF HIV STATUS}

Discussions of disclosure, including being disclosed to and disclosing HIV to others, commonly accompanied discussions of HIV treatment and one's identity with HIV. According to study sampling criteria, all of the participants in the current study were aware of their HIV status. ART clinical guidelines in South Africa dictate that all children should be "disclosed to" as soon as they reach their teens. ${ }^{16}$ The disclosure process is specific. First, the health worker or adherence counsellor gives a booklet to the parent or guardian to read with her/his child. Then, an appointment is set with the parent, child, health worker and social worker, where the child will be informed of her/his HIV status as the reason why s/he is taking medication. One participant, Zukiswa (girl 10), recounted asking her mother about her HIV status, even before the formal process was initiated, indicating that disclosure can happen in different ways. The majority of participants who discussed being disclosed to described it as a positive experience because, as Busi (age 10) put it, she liked knowing the reason for taking the medication.

Accordingly, several participants described their families as consistently supportive in their lives, as Mulelwa (girl 15) captured with an analogy, a picture of a "beautiful sunset that goes up and down but remains present." Overall, outside of their parents or close family members, however, most youth kept their HIV status secret. Mulelwa was actually the only participant who said she lived openly with her HIV positive status at school. She said she attended a support group at school for girls with "HIV or an STI or who are pregnant...I do attend these groups because I already told my friends my status." She depicted her support system with a picture of a tree:

\footnotetext{
"The branches grow in different ways but they always stand together as one tree. It reminds me that although sometimes it's hard to drink pills I have people who can support me to drink them because we are family and we stand together."
}

Others, like Henriette (girl 16), were very selective about who they told. She, for example, had one friend that knew

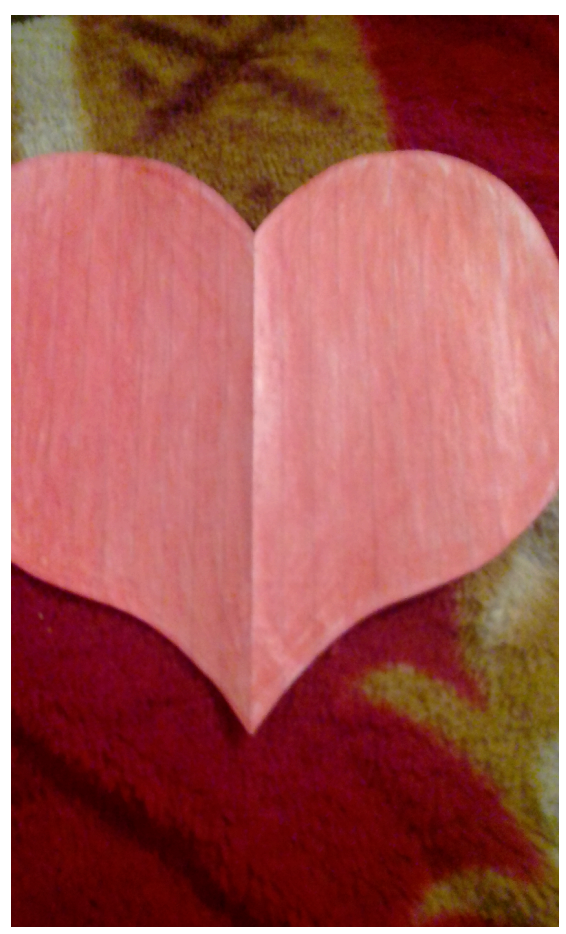

Figure 2. Survivor.

her status. Similarly, Tumi (boy 14) only told his teachers. Bernadette (girl 16) kept her status completely secret from outsiders, and explained that she hid her medication from other members of the household and people who visited her house.

"I put my medicine] in a different pot because people can google [the name of the tablet]. But, everybody has blood. I put my medicine in blood tube and put it in my dad's bag because he has blood and people think it's his."

She also explained that her boyfriend did not know she took medicine even though he was supportive of her emotional health overall.

\section{LIVING CONDITIONS}

In addition to talking about personal aspects of treatment and living with HIV (medication prompts, identity with HIV), and relational experiences of treatment and HIV (disclosing status to others), participants also discussed and captured their living conditions or the context of their treatment and health. As described in previous sections, participants discussed access to clean water and nutritious food as important facilitators of taking HIV medication. Participants connected poverty and housing to their health, and the living conditions of participants in our study were generally not favourable. However, there were exceptions as illustrated by Tuli (boy 15) with the picture that he shared (Figure 3). [He was an African boy who was adopted by White parent, and was currently living with his father in a more affluent neighbourhood.] He photographed a sunset to describe how he kept himself happy with frequent walks on the beach. Tuli, by virtue of being of a higher socioe- 
conomic stratum, also had more opportunities for recreational activities, as illustrated by another picture he took of Table Mountain, which reminded him of the hiking trip he did with friends.

In contrast, the majority of the participants in the current Photovoice study, and by extension most adolescent HIV patients in South Africa, reside in townships where living conditions are poor. When asked about their recreational activities, some participants actually said they did not even go outside. Tumi (boy 14) for example, discussed HIV and HIV treatment by illustrating the way certain neighborhoods facilitated the spread of illness and hindered selfcare (Figure 4).

\begin{abstract}
"I took this picture because I was very...curious about what happened in that place, and also, I was also sad because there are some children who get infected with bad diseases because of places like that...There are places that were burnt and also, some places uh that were pushed by the air [wind]... and had fallen down because [the people who live there don't have money] and use material that is not strong enough to build their homes."
\end{abstract}

Yet, despite the challenging living conditions that are prevailing in townships across the Cape metropolitan area, Tumi expressed hopefulness for the future and a measure of thankfulness for the development that did take place through the following picture (Figure 5):

\begin{abstract}
"This picture makes me happy because it makes me think of the history of this place and also how the economy has grown. I want people to see that there are also people without material but who have emotional support. Some people live in places that are like that, but they are also happy because they are happy to be living than those who are not living."
\end{abstract}

The adolescent girl discussion group discussed another living condition challenge - the lack of adequate transport to reach treatment. Most participants described being dependent on public transport, which presented problems with arriving on time for appointments and meetings. Bernadette, for example, chose to attend clinic and high school in a township that is further than walking distance, and said:

"My fifth picture is about taxis. I must travel to school by taxi. There's no personal transport which is why sometimes I'm late for school - because of taxi's ...today I was late. I stood half an hour by the taxi rank waiting for a taxi. And this time of the day taxis are few. You must grab one when you get one. So, to me like, taxi's is a big problem in my life. Because sometimes they strike then what do you do? Walk and for me to walk to the hospital is very far so I can't walk."

\section{DISCUSSION}

The Photovoice methods were effective in getting most of the adolescent participants to talk freely about their experiences of living with HIV and being on ART. The participants were mostly from the low to lower socioeconomic strata of society, and appreciated the free "cell phone" they received

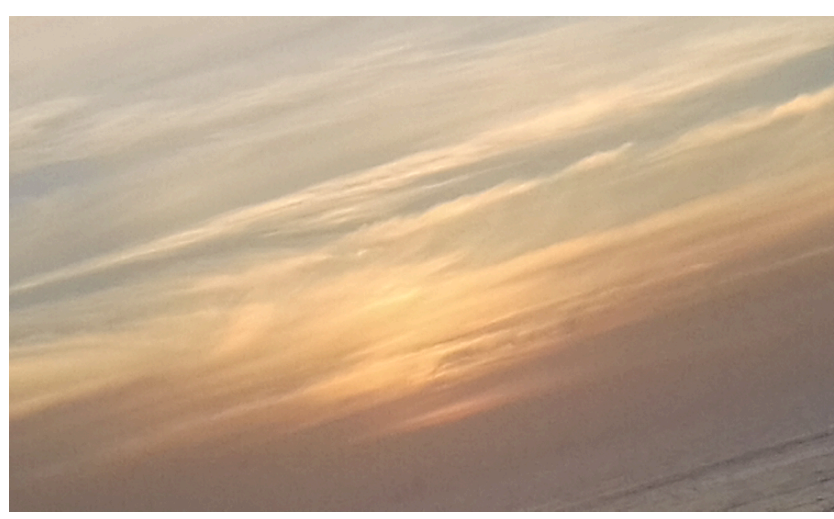

Figure 3. The beach.

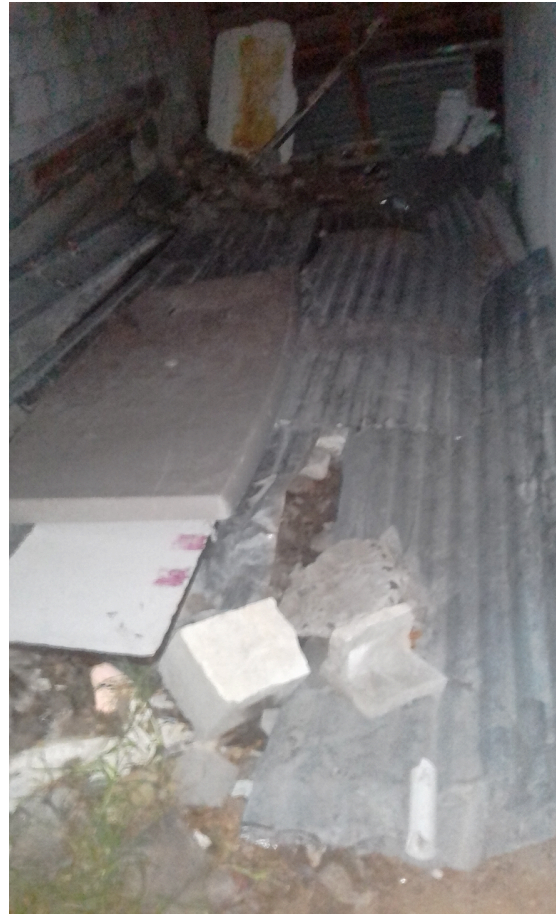

Figure 4. Poor living conditions.

upon completion of the study. The older adolescents revelled in the opportunity to take photos and be creative in sharing their stories. The pilot was also very successful in giving power to the participants to direct the discussion and talking points. Malmqvist et al. ${ }^{15}$ argued that a carefully organized and managed pilot study can increase the quality of the research as results from such studies can inform the main study and subsequent parts of the research. Our pilot study will be followed with a larger qualitative study, as well as an explorative qualitative study on the delivery of HIV care in the health facilities where participants receive treatment. Our pilot study also fulfilled the suggestion by Teti $^{14}$ to rigorously 'explore' the intricacies of the ethics of conducting a Photovoice study with younger adolescents in the South African public health care setting. The youngest participants faced challenges to fully participating in the study. Two 10 year old girls only took two photos each of themselves and their friends and parents. Compared to 
older adolescents, younger adolescents were limited in expressing their experiences through taking photos of objects in their surroundings. In the next study, much clearer instruction will be given to them to take at least five photos and encourage creative thinking.

Resultant themes reflected the participants' positivity towards being on treatment and adhering to their medication. This topic was somewhat expected as all the participants were engaged in care at the time of the research. Their self-reported use of daily reminders to take their treatment, similar to what Van Wyk and David ${ }^{9}$ found, reflected their positive uptake of the treatment literacy and education that they received from the health care workers. The extent to which they learned health education was also reflected in discussions about eating healthy and staying away from drugs and alcohol and tobacco use. This finding resonates with Taukeni and Ferreira's ${ }^{17}$ work that health workers are a key source of information about HIV and AIDS, and can play a vital supporting role in communicating with children.

The abovementioned "positivity" was in the context of challenging socioeconomic and environmental conditions, and family conflict as well as questions about self and stigma. Their photos depicted and promoted discussions about a mentality where they wanted to, or perhaps were coached/ encouraged by health workers to look for positive things in their lives to motivate them to take their treatment. For example, even threats (of dying or being hospitalised) were used to motivate them to continue taking their treatment, and most of the adolescents perceived this as encouraging, and indicative of social support. ${ }^{18}$

Though all participants knew they were HIV positive, there were considerable variations with respect to disclosure to family and friends. Most kept disclosure of their HIV status confined to those who-need-to-know, i.e. those in the household that would provide reminders to take their medications on time. Only one participant disclosed her HIV status outside of her family to a support group at school. Ngeno and colleagues ${ }^{19}$ reported that adolescents that disclosed their HIV status to a peer support group had better adherence and treatment outcomes compared to those who did not.

\section{LIMITATIONS}

This was a pilot study and was subject to several limitations. The sample was small and rather homogenous. For example, all of the participants were from an urban area, and most were struggling with poverty and lack of resources. Themes and photos might be different if participants were in a rural setting, or had access to additional resources. The researchers were also only able to recruit participants who were willing to engage in elements of the project, including taking pictures, sharing their ideas with others, and being open about their HIV status in a small group. Young people who are hiding their HIV status would not be able to take part in the project as constructed; and our findings do not capture the needs or experiences of youth who have not yet shared their HIV status with their peers.

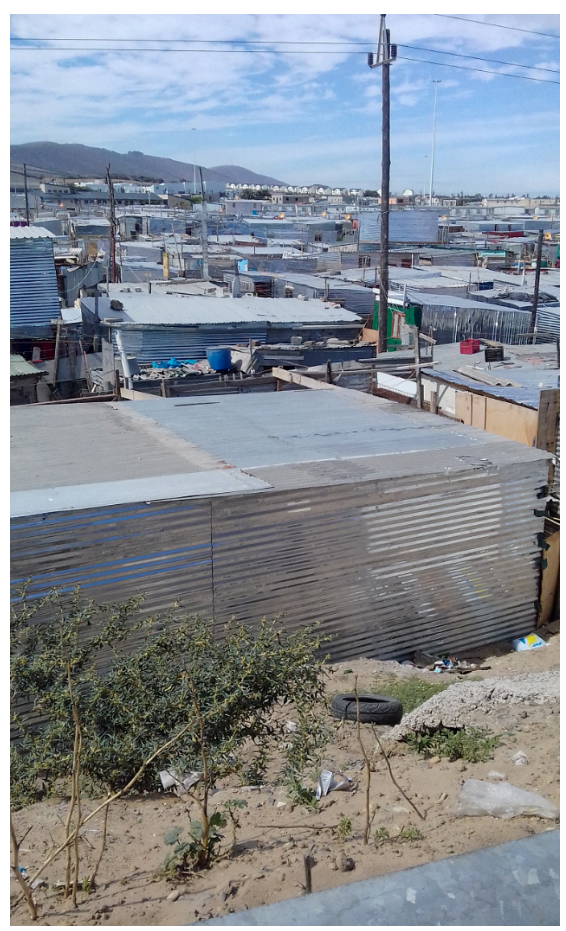

Figure 5. Hope for the future.

\section{CONCLUSIONS}

These limitations notwithstanding, this study resulted in new knowledge about how to adapt Photovoice methods with adolescents, particularly for those under 14 years of age. The pilot showed us that we need to take greater care in giving clear instructions to younger adolescents about what photos to take, and give them more "ideas" of what they can take. At the same time, we also learned that it is best to give older adolescents fewer instructions, so that they can exercise maximum creativity.

Adolescents enjoyed Photovoice methods and felt "empowered" to tell their own stories through the photos. This finding supports future Photovoice research as a valuable way to learn about HIV medication adherence. Photovoice can be a supportive intervention itself, deserving of future testing.

Our findings confirm reports from other studies with ALHIV in similar settings - most notably issues around the value of medication reminders and difficulties around disclosure. Thus, disclosure support - for parents disclosing HIV to their children, and for youth to safely disclose their status to others, is still needed. Our study findings also indicate that adolescent participants desired acceptance by the health services personnel (and the researchers, by extension) by reflecting back what they were told in health education and treatment literacy sessions. This desire for acceptance could be a building block for interventions to support adherence and retention in care by providing psychosocial support in conjunction with clinical care. Although youth felt supported by messages they received from others, the threatening nature of the messages indicates that there may be room to assist parents or caregivers to develop new and less threatening forms of motivation. 
Acknowledgments: The views expressed in the submitted article are the authors and not an official position of the institutions or funder. The authors have no conflicts of interest to declare. This study was approved by the primary author's institutional research board.

Funding: This work was funded by the South African Medical Research Council under a Self-Initiated Research grant.
Authorship contributions: B.V.W. and M.T.

Competing interests: The authors completed the Unified Competing Interest form at www.icmje.org/coidisclosure.pdf, and declare no conflicts of interest.

\section{Correspondence to:}

Michelle Teti

University of Missouri Department of Public Health School of Health Professions

Columbia, Missouri, MO 65201, United States tetim@health.missouri.edu 


\section{REFERENCES}

1. UNAIDS. AIDSinfo. UNAIDS 2019 estimates. 2019. https://www.aidsinfo.unaids.org.

\section{World Health Organization [WHO]. Global} Accelerated Action for the Health of Adolescents (AAHA!): Guidance to Support Country Implementation. Summary. Geneva: WHO; 2017. http://www.who.int/ maternal_child_adolescent/topics/adolescences/frame work-accelerated-action/en.

3. Kung TH, Wallace ML, Snyder KL, et al. South African healthcare provider perspectives on transitioning adolescents into adult HIV care. $S$ Afr Med J. 2016;106(8):804. doi:10.7196/samj.2016.v106i $\underline{8.10496}$

4. Cervia JS. Easing the Transition of HIV-Infected Adolescents to Adult Care. AIDS Patient Care and STDs. 2013;27(12):692-696. doi:10.1089/apc.2013.025 3

5. Pinzón-Iregui MC, Ibanez G, Beck-Sagué C, Halpern M, Mendoza RM. ...like because you are grownup, you do not need help": Experiences of transition from pediatric to adult care among youth with perinatal HIV infection, their caregivers, and health care providers in the Dominican Republic. Journal of the International Association of Providers of AIDS Care. 2017;16(6):579-587. doi:10.1177/23259 $\underline{57417729749}$

6. Vijayan T, Benin AL, Wagner K, Romano S, Andiman WA. We never thought this would happen: Transitioning care of adolescents with perinatally acquired HIV infection from pediatrics to internal medicine. AIDS Care. 2009;21(10):1222-1229. doi:1 $\underline{0.1080 / 09540120902730054}$

7. Machado DM, Succi RC, Turato ER. Transitioning adolescents living with HIV/AIDS to adult-oriented health care: An emerging challenge. J Pediatr (Rio J). 2010;86(6):465-472. doi:10.2223/iped.2048

8. Mark D, Armstrong A, Andrade C, et al. HIV treatment and care services for adolescents: A situational analysis of 218 facilities in 23 sub-Saharan African countries. Journal of the International AIDS Society. 2017;20:21591. doi:10.7448/ias.20.4.21591

9. Van Wyk BE, Davids L-AC. Challenges to living with HIV and adherence to antiretroviral treatment amongst school-going adolescents in a low socioeconomic urban setting in Cape Town, South Africa: a qualitative study. South Afr j HIV med. 2019;20(1). do i:10.4102/sajhivmed.v20i1.1002
10. Kahana SY, Rohan J, Allison S, Frazier TW, Drotar D. A meta-analysis of adherence to antiretroviral therapy and virologic responses in HIV-infected children, adolescents, and young adults. AIDS Behav. 2013;17(1):41-60. doi:10.1007/s10461-012-0159-4

11. Petersen I, Bhana A, Myeza N, et al. Psychosocial challenges and protective influences for socioemotional coping of HIV+ adolescents in South Africa: A qualitative investigation. AIDS Care. 2010;22(8):970-978. doi:10.1080/09540121003623693

12. Larkan F, van Wyk B, Stevens P, Saris AJ. Between the clinic and the community: Temporality and patterns of ART adherence in the Western Cape province, South Africa. African Sociological Review. 2015;19(2):26-52.

13. Azia IN, Mukumbang FC, Van Wyk B. Barriers to adherence to antiretroviral treatment in a regional hospital in Vredenburg, Western Cape, South Africa. South Afr j HIV med. 2016;17(1). doi:10.4102/sajhivm ed.v17i1.476

14. Teti M. The murky ethics of visual qualitative methods: Picturing a clear path forward. International Journal of Qualitative Methods. 2019;18:1-4. doi:10.1177/16090406919884810

15. Malmqvist J, Hellberg K, Möllås G, Rose R, Shevlin M. Conducting the pilot study: A neglected part of the research process? Methodological findings supporting the importance of piloting in qualitative research studies. International Journal of Qualitative Methods. 2019;18:160940691987834. doi:10.1177/16094069198 $\underline{78341}$

16. National Department of Health. National Consolidated Guidelines for the Prevention of Mother-to-Child Transmission of HIV (PMTCT) and the Management of HIV in Children, Adolescents and Adults. Pretoria: National Department of Health; 2015 .

17. Taukeni S, Ferreira R. HIV and/or AIDS awareness among adolescents in a South African at-risk rural community. South Afr j HIV med. 2016;17(1). doi:10.4 102/saihivmed.v17i1.418

18. Gentz SG, Calonge-Romano I, Martínez-Arias R, Zeng C, Ruiz-Casares M. Mental health among adolescents living with HIV in Namibia: The role of poverty, orphanhood and social support. AIDS Care. 2018;30(sup2):83-91. doi:10.1080/09540121.2018.146 9727 
19. Ngeno B, Waruru A, Inwani I, et al. Disclosure and clinical outcomes among young adolescents living with HIV in Kenya. Journal of Adolescent Health.

2019;64(2):242-249. doi:10.1016/j.jadohealth.2018.0

$\underline{8.013}$ 